Volume 9, No.1.3, 2020

International Journal of Advanced Trends in Computer Science and Engineering

Available Online at http://www.warse.org/IJATCSE/static/pdf/file/ijatcse3691.32020.pdf

https://doi.org/10.30534/ijatcse/2020/3691.32020

\title{
Automated Sequential Controller for Pre-Seal Bake Process of Microelectronic Packages
}

\author{
Raymundo P. Gamboa ${ }^{1}$, Glenn N. Ortiz ${ }^{2}$, Mark Joseph B. Enojas ${ }^{3}$ \\ ${ }^{1}$ Analog Devices Inc., General Trias Cavite, Philippines, rhed87@ gmail.com \\ ${ }^{2}$ Technological University of the Philippines, Taguig City, Philippines, glenn.ortiz46@yahoo.com \\ ${ }^{3}$ Technological University of the Philippines, Taguig City, Philippines, markjoseph_enojas@tup.edu.ph
}

\begin{abstract}
This study presents an automated process and setup for pre-seal bake of microelectronic packages. It reduces the tendencies of error for critical temperature and vacuum pressure control during the pre-seal bake. The new suggested automated process delivers a faster and more reliable system than that of the presented case of a semi-automated pre-seal bake process. The automated process is an integration of ADC (analog-to-digital converter), opto-isolator, signal conditioning devices, and microcontroller. An average of 1 hour and 31 minutes for the whole process to complete has been recorded from multiple tests. The operator engagement was reduced to 10 minutes from a 51-minute routine. Additionally, since the automated process lessens the operator engagement, thus human error is also reduced. This automated process is proven to improve the system of pre-seal bake process.
\end{abstract}

Key words: automated sequential controller, gas bake, microelectronic packages, opto-isolator, vacuum bake, pre-seal bake process.

\section{INTRODUCTION}

\subsection{Microelectronic Packaging Trends}

The microelectronic packaging technology research and developments focus on different areas such as materials and its processing, processes and its automation, designs, modeling, simulations, and higher reliability [1]. These areas play major roles in the progress of development and innovation of microelectronic packages. Furthermore, applications to different fields have made such microelectronic packages come to an evolution. Some fields of sciences and engineering such as medical, telecom, computer, aerospace, automotive, consumer, education, manufacturing, and etc., pave the way for massive development of these products [2].
Different substrates are being added to microelectronic packages in order to achieve better product quality. These packages should withstand harsh plant environment. High reliability demands need high susceptibility to such environments for these packages. A study on moisture sensitivity level of microelectronic packages were conducted with different methods such as equal weight and equal moisture distribution [3-4]. Automation in moisture analysis has also been conducted to address such critical parameter [5-6] using simulations in finite element workbenches and functional elements [7]. There also have been cases reported about the occurrence of high thermal gradients in the manufacturing process of microelectronic packages which results to stresses in the products [8]. However, other packages operate in low temperatures because of the type of materials being used such as thick film copper and carbon nanotubes (CNTs) [9-11]. Therefore, there is a need in the production to closely monitor and control such parameters. Other parameters such as electrostatic discharges are considered in the production in which protection is needed [12].

Different processes also exist in different packages. In one of the euro-dots course program presented [13], three different levels of microelectronic assembly has been presented. Level one is the wire bonding, second, is the assembly of different packages in PCBs, and the third level is the motherboard. The first level also involves control of temperature and pressure. Even test chips for microelectronic packages also needs proper monitoring and control of temperature and pressure in its production process [14]. This study addresses one of the processes in the production of microelectronic packages as a vital parameter to increase reliability [15].

\subsection{Pre-seal Bake Process}

Pre-bake seal process is the initial stage of special procedure on preparing the microelectronic products before being processed in the production area. This process is done to remove moisture from the material before going further from one station to another. The pre-seal bake oven evacuates the product automatically at specific vacuum level, bake time, and temperature at a certain period. The manual purging of 
gas to the oven to set the pressure parameters prior to unloading are done. (See Fig. 1). These ovens are equipped with calibrated temperature and vacuum pressure controllers. Pre-seal bake process for microelectronic packages typically needs monitoring of pressure and temperature inside the oven [15]. The microelectronic units are subjected to a certain heat and pressure requirements. There is susceptibility to error when these process are done manually which results to downtime and rejects. For high precision products, low power requirements need critical designs and processes, thus need to be considered in automation.

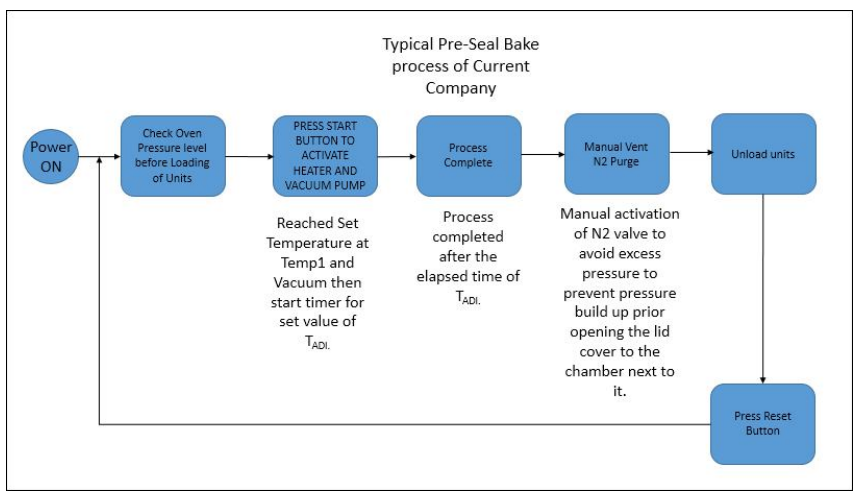

Figure 1: Existing Pre-Seal Bake Process Flow Diagram

\subsection{Automation Needs for Pre-Seal Bake Process}

As aimed in the long run by developers and researchers, the automated sequential controller would have the advantage of reduce thermal stress, increase reliability, packaging cost reduction, and cutting production time and errors [16] in the pre-seal bake process of microelectronic packages. In microelectronic packaging, automation is designed and developed from the production to the testing of products and equipment [17].

A new process for pre-seal bake has been introduced which requires Gas bake and Vacuum bake that are set in certain temperature and pressure. This new process requires gas evacuation and purge between the gas bake and vacuum bake for unloading. Such limitations of these manual processes has to be addressed through automation. In this paper, an automated sequential controller was introduced in the pre-seal bake process of microelectronic packages without interruption to the existing setup of pre-seal bake to avoid overhauling or change of machine and processes. This is done through a process mode selection switch, as presented in Fig. 2.

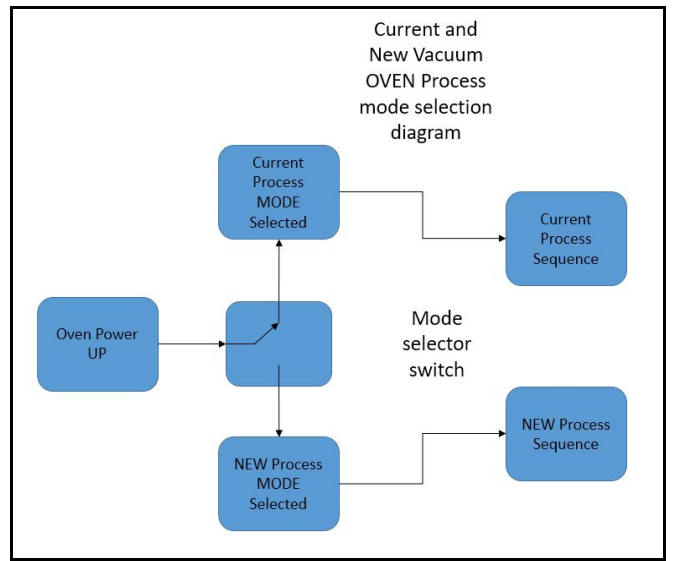

Figure 2: Process Mode Selection Diagram

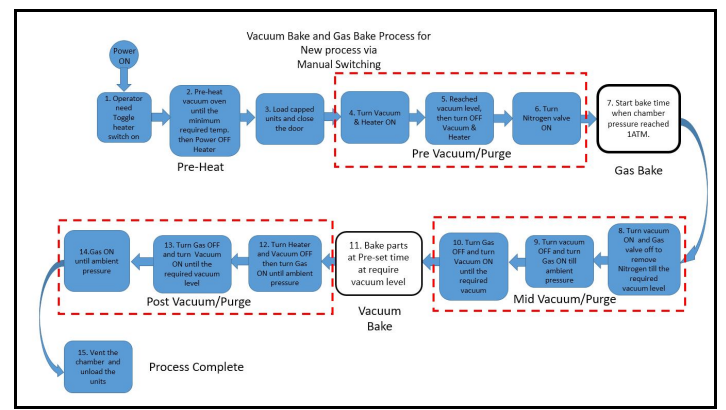

Figure 3: Improved Pre-Seal Bake Process Flow Diagram

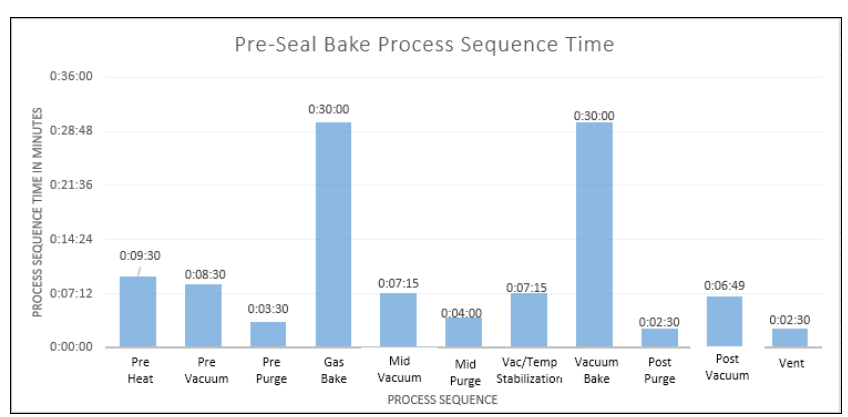

Figure 4: New Product Sequence Time

In the new process flow, the following sequence is being automated: pre-heat, pre-vacuum, pre-purge, mid-vacuum, vacuum/temperature stabilization, post-purge, post-vacuum, and venting. A case study was conducted in a microelectronic package manufacturer in their pre-seal bake process with the following operator engagement time recorded and presented in Table 1. In average, with the operator engagement, the complete cycle on the pre-seal bake process takes 2 hours and 12 minutes.

Table 1: Operator Engagement Time

\begin{tabular}{|l|l|}
\hline \multicolumn{1}{|c|}{ Sequence } & Time (minutes: seconds) \\
\hline Pre-heat & $9: 30$ \\
\hline Pre-vacuum & $8: 30$ \\
\hline Pre-purge & $3: 30$ \\
\hline Mid-vacuum & $7: 15$ \\
\hline Mid-purge & $4: 00$ \\
\hline
\end{tabular}




\begin{tabular}{|l|l|}
\hline \multicolumn{1}{|c|}{ Sequence } & \multicolumn{1}{c|}{ Time (minutes: seconds) } \\
\hline Vacuum/Temperature Stabilization & $7: 15$ \\
\hline Post-purge & $2: 30$ \\
\hline Post-Vacuum & $6: 49$ \\
\hline Vent & $2: 30$ \\
\hline Total & $\mathbf{5 1 : 4 9}$ \\
\hline
\end{tabular}

\section{METHODOLOGY}

\subsection{Process Flow}

The automated process sequence is integrated to the former manual system such that a selector switch toggles this option. (See Fig. 5). The units will be loaded into the chamber after the oven pre-heating is done. The temperature and pressure settings are based on the product specification and are controlled by the built in temperature and vacuum pressure controllers.

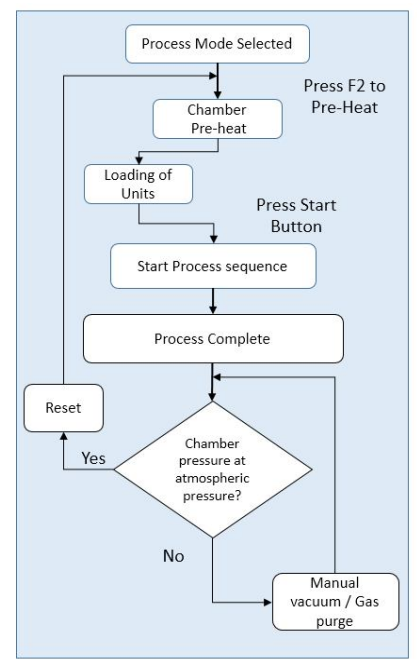

Figure 5: Automated Sequential Controller for Pre-Seal Bake Process Flow

\subsection{System Design}

The automation system is composed of the control panels, $\mathrm{ADC}$ and DAC, temperature sensors and vacuum pressure sensors as inputs, microcontroller unit as the main controller, temperature controllers, vacuum pressure controllers, and output devices such as the heater, vacuum pump, and solenoid valves. The system runs in a feedback control loop. (See Fig. 6). Important parts are being broken down and explained as follows:

1. Keypad - A modified one-wire keypad is used as an interface for setting the temperature and pressure parameters.

2. Feedback signals - It is noted that the feedback signals are isolated via opto-isolator because of its high AC potential to protect the controllers. The gas valve is driven by the temperature controller to maintain it in desired levels.
3. Power system - the input and output devices runs in different power levels. In this case, external power sources are needed such as in valves which runs in $24 \mathrm{~V}$ power. On the other hand, some most input devices have signals ranging from 3.3-5.0 V. (See Fig. 7)

4. Temperature signal conditioning transmission - the temperature sensor used for measurement and monitoring is a type $\mathbf{J}$ thermocouple. The signals are filtered and amplified as presented in the temperature module in Fig. 8.

5. PCB design - the layout was designed based such that heat emission from other components may not affect other components. (See Fig. 9)

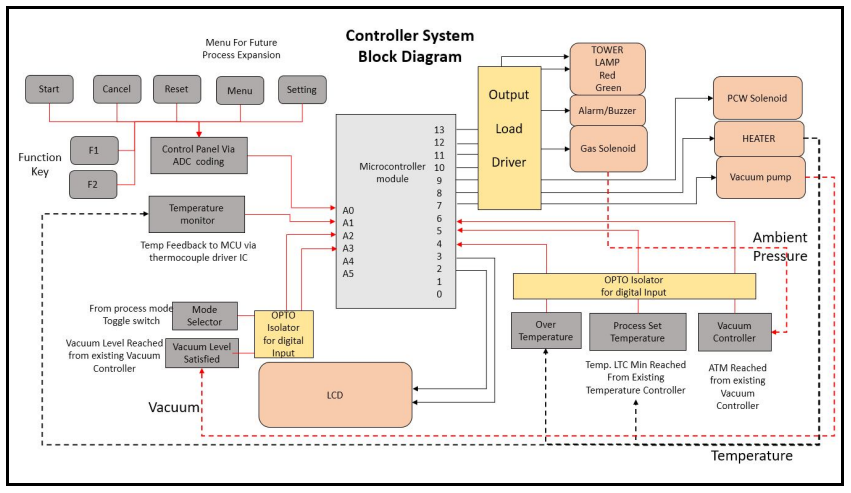

Figure 6: System Block Diagram

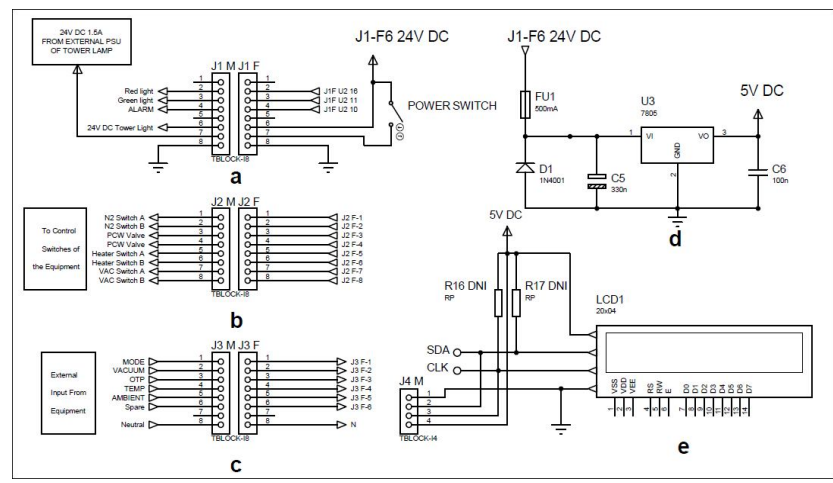

Figure 7: System Schematic Diagram. a) power and tower lamp connections, b) output to toggle switch connectors, c) input devices connectors, d) 5V DC regulator, e) LCD connection

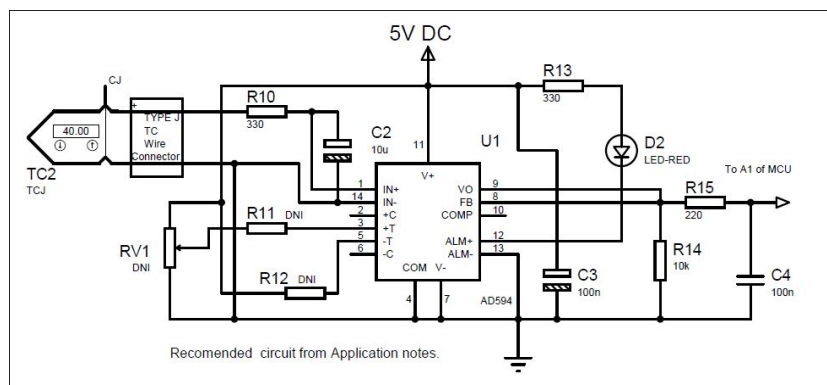

Figure 8: Temperature Signal Conditioning and Transmission 


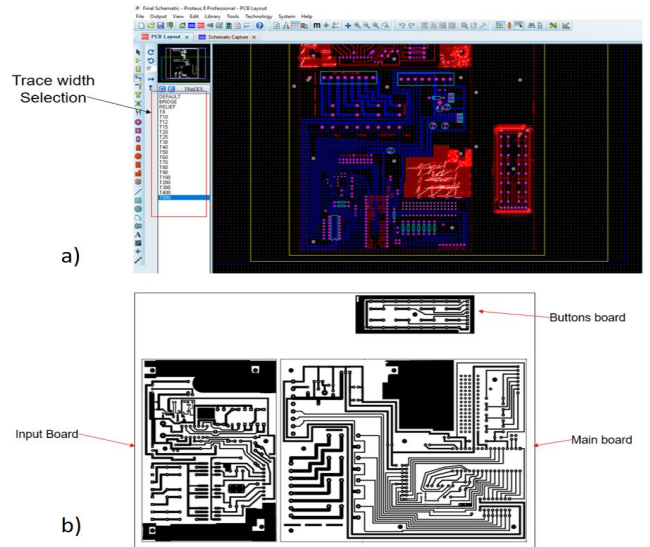

Figure 9: PCB Design. a) Proteus Ares Schematic and PCB design software, b) layout.

\subsection{Tests and Validation}

The prototype is tested and validated based on its functionality. To evaluate the automated system, the improved processing time and error will be compared to that of the former system.

\section{RESULTS AND DISCUSSIONS}

\subsection{Technical Design Output}

Each new product or unit being fed into the pre-seal bake process differs in temperature and vacuum pressure settings. These settings can be adjusted by selection switches and control buttons (See Fig. 10). The control panel buttons have assigned analog input and converted to digital pulses designed with different voltage levels by varying filter circuits.

The modified pre-seal bake oven control sequence is presented in Fig. 12. To isolate the two modes of process sequence, two relays, A1 and A2, are used. Relay A1 used to switch between the former system, mode A, and the automated system, mode B. Relay A2 is used to switch the connection output relays of vacuum controller, temperature controller, and timer in the two modes that provides feedback input to the automated sequential controller for pre-seal bake process. (See Fig. 13).

The temperature feedback circuit monitors the temperature during gas purging to ensure that the oven temperature will not exceed to the required maximum temperature. A PID controller was used, utilizing type $\mathrm{J}$ thermocouple as the input device. An Analog Devices Inc. product, AD594, which has an accuracy of +1 to +3 . was used as the thermocouple driver. It is enclosed in 14-lead ceramic package which is powered by a single ended supply.

To reduce AC noise in the non-inverting input of the instrumentation amplifier, a low-pass filter is used. The output signal is also filtered at a maximum frequency of $60 \mathrm{~Hz}$. The output device has a resolution of $10 \mathrm{mV} /{ }^{\circ} \mathrm{C}$ at a range of $5 \mathrm{~V}$. The conversion formula used is (1), where $\mathrm{V}_{\text {in }}$ is the input voltage and $\mathrm{V}_{\text {ref }}$ is the voltage reference.

$$
A D C=\frac{1023 V_{i r}}{V_{r q f}}
$$

A multi-channel input opto-isolator ILD620 which uses parallel GaAs IRLED emitter and high gain NPN silicon phototransistors (Vishay) per channel that can withstand $5300 \mathrm{Vrms}$ test voltage are used in the AC input. The peak voltage of the AC input for the proper current limiting resistor computation is needed. Eq. (2) is used to convert the peak voltage.

$$
V_{p}=\sqrt{2} V_{i n}
$$

To reduce AC noise in the non-inverting input of the instrumentation amplifier, a low-pass filter is used. The output signal is also filtered at a maximum frequency of $60 \mathrm{~Hz}$. The output device has a resolution of $10 \mathrm{mV} /{ }^{\circ} \mathrm{C}$ at a range of $5 \mathrm{~V}$. The conversion formula used is Eq. 1, where $\mathrm{V}_{\text {in }}$ is the input voltage and $\mathrm{V}_{\text {ref }}$ is the voltage reference. Several trials have been conducted and the final optimized input design was presented in Fig. 14.

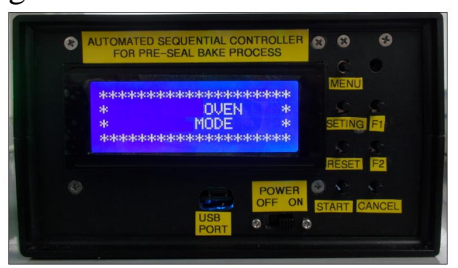

Figure 10: Actual prototype of the automated sequential controller for pre-seal bake process

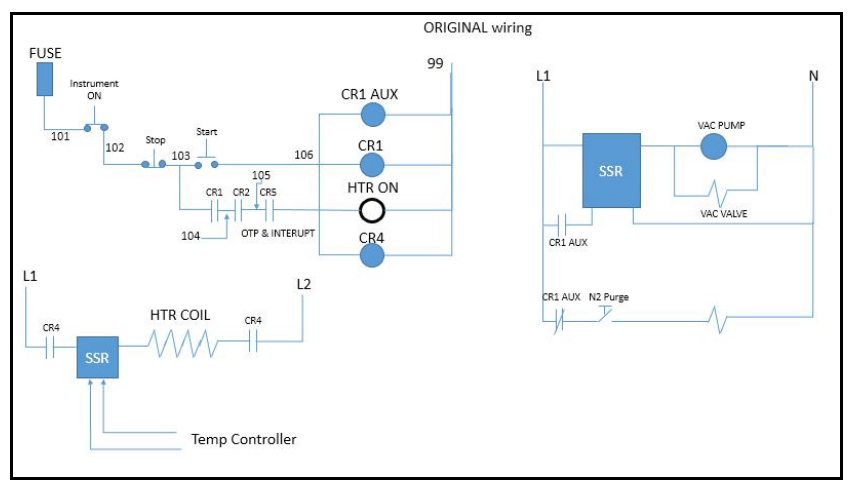

Figure 11: Pre-Seal bake oven wiring diagram

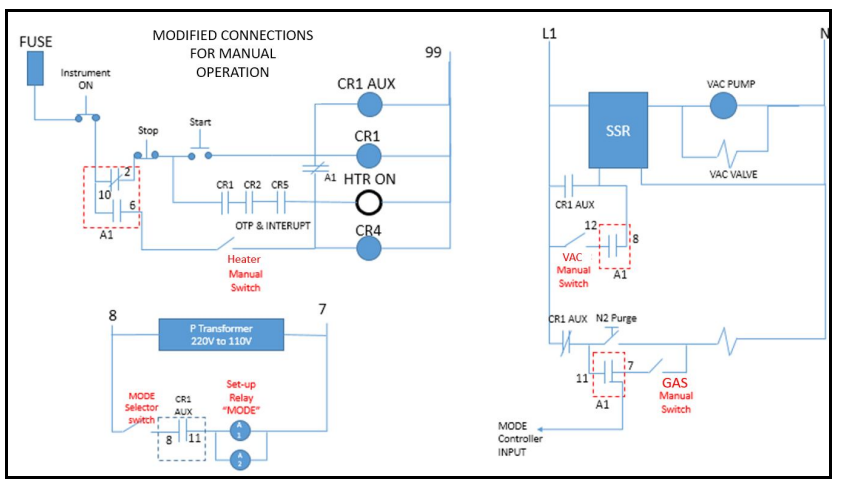

Figure 12: Modified pre-seal bake oven control wiring diagram 


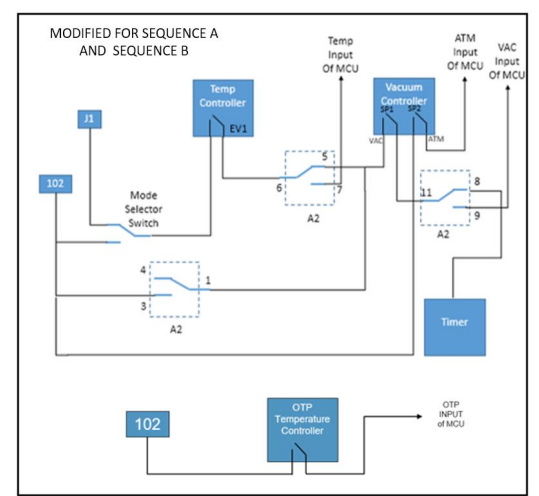

Figure 13: Modified output relay connections of vacuum controller, temperature controller, OTP temperature and process timer

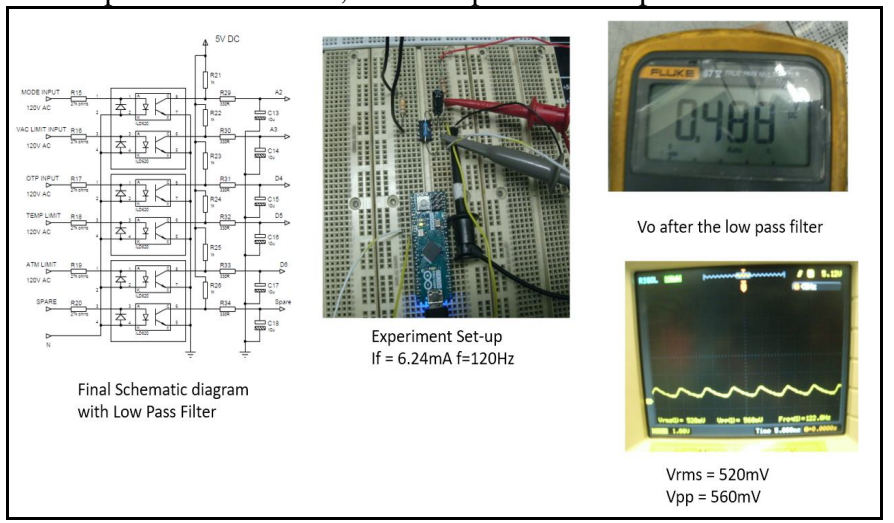

Figure 14: Input schematic diagram, experimental setup, and actual voltage and signal output results

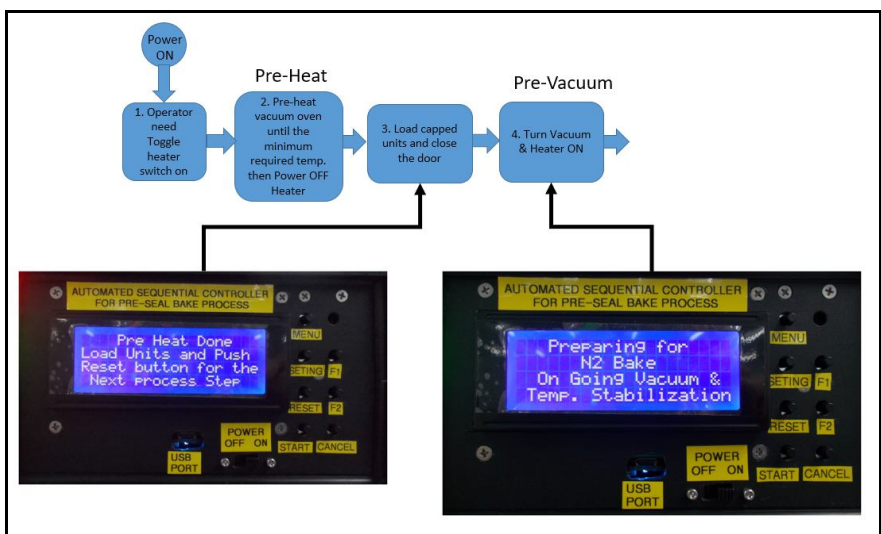

Figure 15: LCD display for pre-heat and pre-vacuum completion

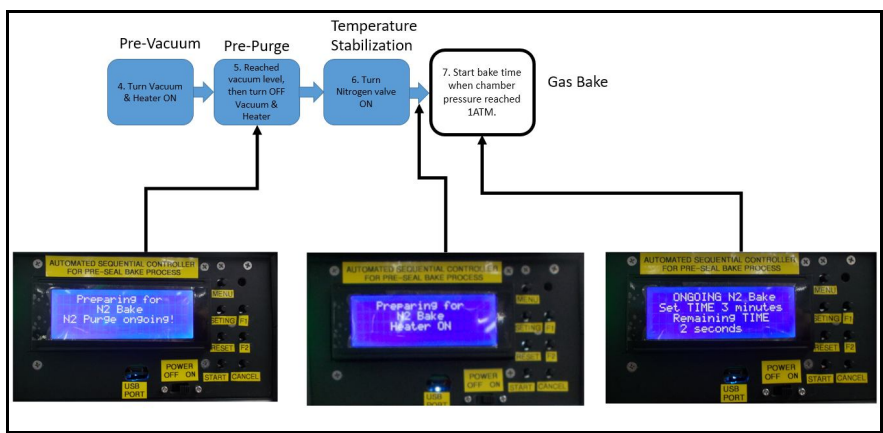

Figure 16: Pre-purge and temperature stabilization

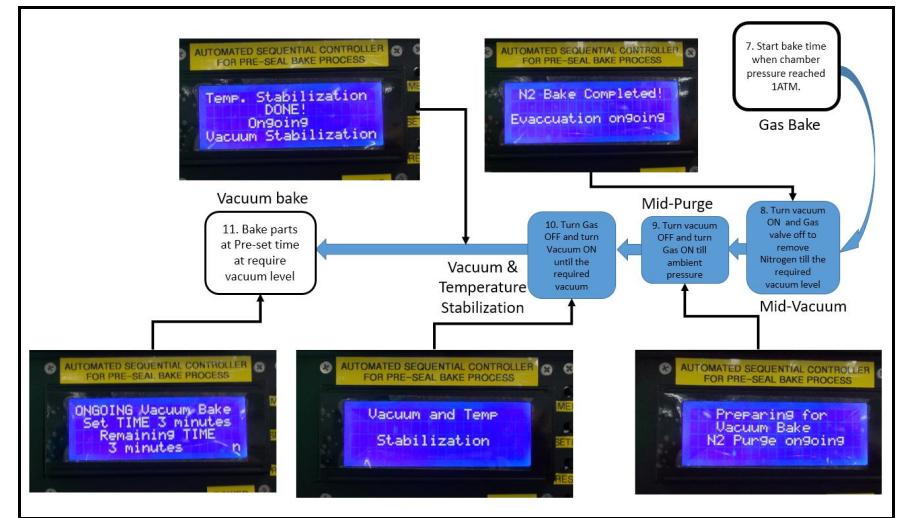

Figure 17: Mid-vacuum/purge and post-vacuum/purge

\subsection{Functionality test results}

The functionality of the system was tested accordingly by loading different units for the pre-seal bake process. A toggle switch to turn on the heater. At this point, the temperature controller will function until the desired temperature is met. After the preheating, the units will be loaded in the oven. Pre-vacuum process comes next. The response of the system is presented in Fig. 15. When the pre-vacuum process is done, both the heater and the vacuum will be turned off and nitrogen is being purged into the system for temperature stabilization. Once the initialization is done, the pre-seal gas bake will start. (See Fig. 16). At the mid-vacuum/purge, the vacuum will be turned on while the gas valve will be turned off. The nitrogen at this stage will be removed to an amount until the set vacuum level is achieved. At mid-purge stage, the vacuum will be turned off and the gas valve will be turned on once again until the ambient pressure is achieved. These processes are presented in Fig. 17.

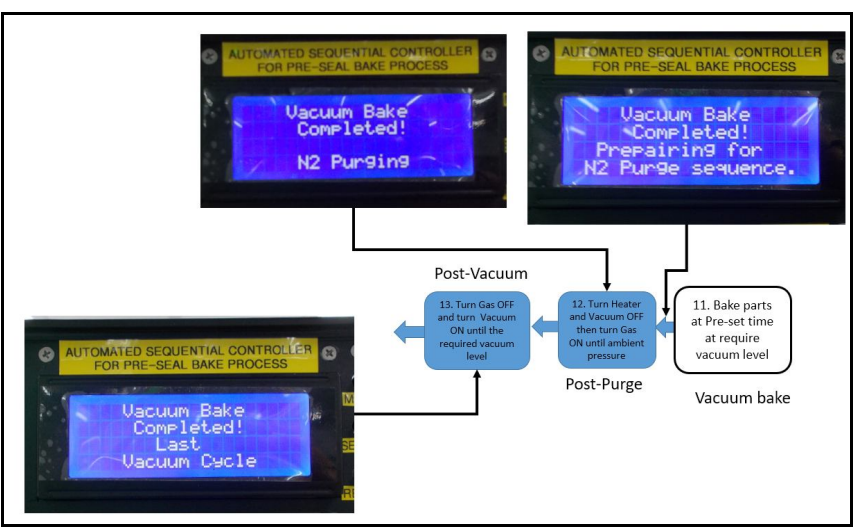

Figure 18: Post-purge and post-vacuum output 


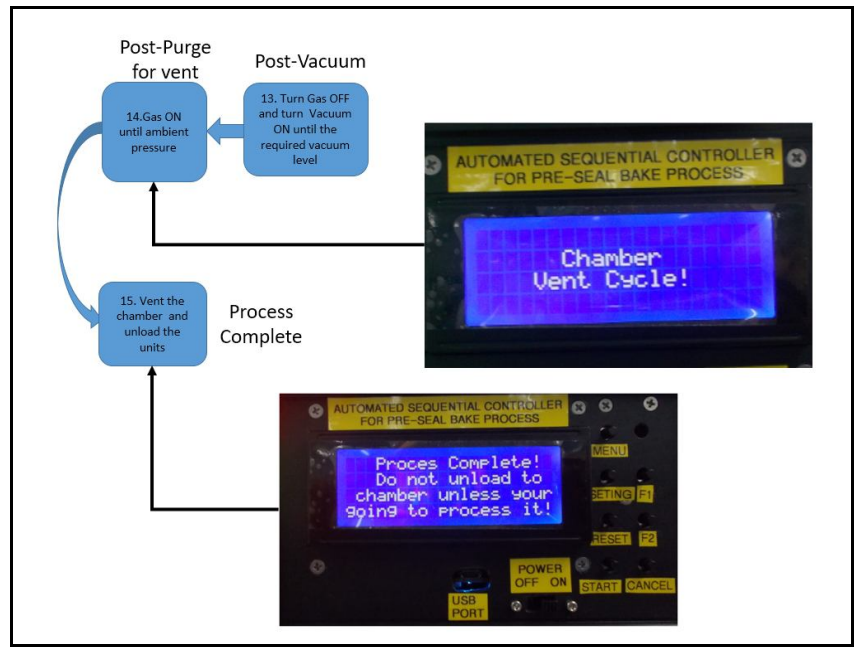

Figure 19: Post-purge for vent to complete the whole process

Another run of turning of the gas valve and turning on the vacuum will be repeatedly done until the vacuum and temperature levels stabilizes. Once this is achieved, vacuum will be performed next. Once the vacuum bake is completed, the post-purge is performed such that the heater and the vacuum will be turned off and the gas valve will be turned on until the set ambient pressure is met.

Post-vacuum process comes next when the parameters stabilizes and the gas valve is turned off and the heater and the vacuum valves are turned on. To complete the whole process, these cycles are repeated done until it the post-purge for venting of the chamber is reached to unload the units. (See Fig. 19).

A notification will be displayed in the LCD monitor with the confirmation message that the process is already complete. (See Fig. 20). Several runs have been conducted with several microelectronic units loaded in the chamber. The summary of the process time for each stage is presented in Table 2 . The overall cycle time for the four test runs are summarized in Table 3. In the new process sequence, there is no need for user interaction after initiating the pre-seal bake process. The total average cycle time of 1 hour and 31 minutes has been recorded in running the fully automated sequence. The operator engagement takes only 10 minutes to compared to the former which runs in 51-minute routine.

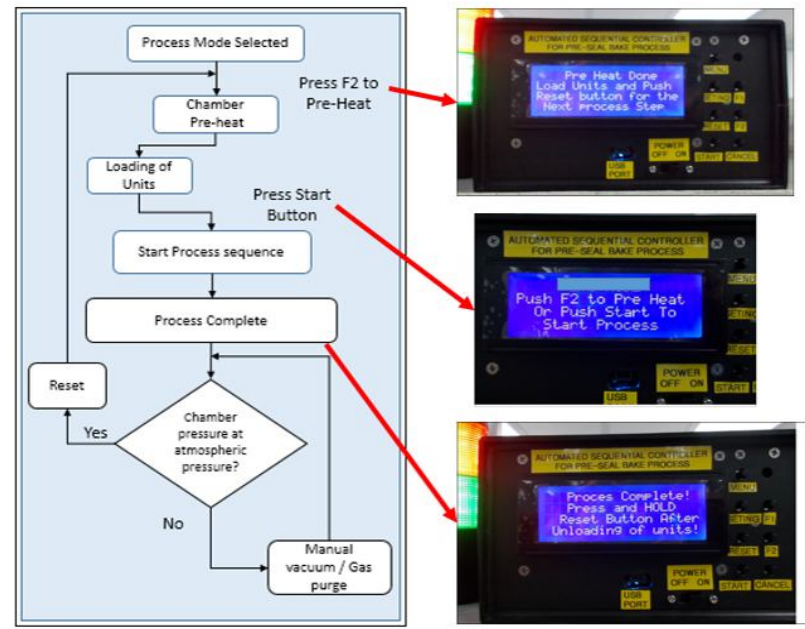

Figure 20: Actual result of completed sequence

Table 2: Stabilization Time with Automatic Sequential Controller

\begin{tabular}{|c|c|c|c|c|c|c|c|c|c|c|c|}
\hline \multicolumn{10}{|c|}{ Per Sequence Stabilization Time using Automatic Sequential Controller } \\
\hline $\begin{array}{c}\text { Evalation } \\
\text { Run }\end{array}$ & Pre Heat & $\begin{array}{c}\text { Pre } \\
\text { Vacuum }\end{array}$ & Pre Purge & Gas Bake & $\begin{array}{c}\text { Mid } \\
\text { Vacuum }\end{array}$ & $\begin{array}{c}\text { Mid } \\
\text { Purge }\end{array}$ & $\begin{array}{c}\text { Vaccuum / } \\
\text { Temperaure } \\
\text { Stabilization }\end{array}$ & $\begin{array}{c}\text { Vacuum } \\
\text { Bake }\end{array}$ & $\begin{array}{c}\text { Post } \\
\text { Purge }\end{array}$ & $\begin{array}{c}\text { Post } \\
\text { Vacuum }\end{array}$ & $\begin{array}{c}\text { Vent } \\
\text { cycle }\end{array}$ \\
\hline 1 & $7: 04$ & $7: 20$ & $2: 04$ & $30: 05$ & $4: 13$ & $1: 38$ & $4: 04$ & $30: 06$ & $6: 02$ & $4: 27$ & $1: 38$ \\
\hline 2 & $6: 52$ & $6: 15$ & $3: 10$ & $30: 42$ & $6: 57$ & $1: 33$ & $5: 36$ & $30: 54$ & $2: 41$ & $5: 09$ & $1: 23$ \\
\hline 3 & $8: 22$ & $4: 25$ & $5: 15$ & $30: 37$ & $3: 29$ & $1: 19$ & $3: 31$ & $30: 41$ & $7: 07$ & $3: 54$ & $1: 21$ \\
\hline 4 & $7: 50$ & $4: 36$ & $4: 01$ & $30: 18$ & $3: 47$ & $1: 18$ & $3: 46$ & $30: 09$ & $7: 10$ & $4: 06$ & $1: 21$ \\
\hline
\end{tabular}

Table 3: New Process Sequential Time

\begin{tabular}{|c|c|c|}
\hline Evaluation Run & Pre-heat & New process cycle time \\
\hline 1 & $7: 04$ & $1: 31: 37$ \\
\hline 2 & $6: 52$ & $1: 34: 20$ \\
\hline 3 & $8: 22$ & $1: 31: 39$ \\
\hline 4 & $7: 50$ & $1: 30: 32$ \\
\hline
\end{tabular}

\section{CONCLUSION}

This study presented a method of automating the pre-seal bake process of a variety of microelectronic packages. In order to prevent over-baking of the product, the temperature control has been automated and optimized. This system is an integration of ADCs, opto-isolators, microcontroller units, and signal conditioning devices. With the automated system, a cycle time of 1 hour and 31 minutes, where the operator engagement is reduced to only 10 minutes, are great advantages in the production which the new automated system has achieved. The operator engagements have been reduced also. The automated system is integrated with the old system so that there will be no interruption to the process. Thus, the old system can still be used as a manual control system and or as bypass whenever necessary. Future works can be conducted such as adding features like wireless remote sensing and monitoring without interrupting the old system.

\section{ACKNOWLEDGEMENT}

This research is conducted in collaboration between Analog Devices Inc., General Trias Cavite and the Technological University of the Philippines Taguig under the 
Raymundo P. Gamboa et al., International Journal of Advanced Trends in Computer Science and Engineering, 9(1.3), 2020,240 - 246

Industry-Based Program. The researchers acknowledge the Lord Jesus Christ for making all these things possible with His guidance and provision.

\section{REFERENCES}

1. W. T. Chen, A. Tseng, P. Way, and S. Clara, "Overview of Recent Developments in Microelectronic Packaging," vol. 00, no. C, pp. $15-16,2001$.

2. F. Drive, "Microelectronic Packaging and Assembly Roadmap for Hong Kong and Pearl River Delta Region: A Team Study and Recommendations," pp. 21-23, 2000.

3. Z. Liu, X. Ma, Q. Ding, and G. Q. Zhang, "Fast MSL Analysis of Microelectronic Packages by using Equal Weight Increasing Method," 2016 17th Int. Conf. Electron. Packag. Technol., no. 1, pp. 391-394, 2016. https://doi.org/10.1109/ICEPT.2016.7583161

4. Q. Ding, X. Ma, and Z. L. G. Q. Zhang, "Fast Analysis and Implementation of Microelectronic Packaging MSL with Equal Moisture Distribution Method," 2016 17th Int. Conf. Electron. Packag. Technol., no. L, pp. 356-360, 2016.

5. Y. Xia, Y. Zhang, L. Liang, Y. Liu, S. Irving, and T. Luk, "Development of moisture automation analysis system for microelectronic packaging structures," 2008 Int. Conf. Electron. Packag. Technol. High Density Packag., pp. 1-7, 2008.

6. S. Sun, J. Song, and Q. Huang, "A compact system-level simulation method for modern microelectronic packaging," 2010 11th Int. Conf. Electron. Packag. Technol. High Density Packag., no. 1, pp. 74-76, 2010.

7. J. Long, "Automated Mechanical Simulation System for Microelectronic Packaging," 2015 16th Int. Conf. Electron. Packag. Technol., pp. 51-55, 2015.

8. C. Basaran and Y. Wen, "Analysis of Multilayered Microelectronic Packaging," vol. 29, no. 4, pp. 850-855, 2006.

https://doi.org/10.1109/TCAPT.2006.885966

9. Z. Xu, Z. Han, Y. Wang, L. Huang, and X. Tang, "Research of Thick Film Copper Circuit in Microelectronic Packaging," pp. 424-426, 2000.

10. K. Zhang and M. M. F. Yuen, "Heat Spreader with Aligned CNTs Designed for Thermal Management of HB-LED Packaging and Microelectronic Packaging," pp. 1-4, 2006.

11. M. Duhan and H. Saini, "Study and Analysis of Low Voltage Low Power Pipelined ADC," International Journal of Advanced Trends in Computer Science and Engineering, vol. 2, no. 2278, pp. 2012-2014, 2013.

12. P. S. Kumar, "Design of Low Power Digital I/O Cell with ESD Ciruit Protection,", International Journal of Advanced Trends in Computer Science and
Engineering, vol. 3, no. 1, pp. 322-325, 2014.

13. H. Debeda, I. Favre, A. Gracia, N. Labat, B. Plano, and $\mathrm{H}$. Fremont, "A one week-lecture in the Euro-dots course program: Microelectronic assemblies: from packaging to reliability," 2013 24th EAEEIE Annu. Conf. (EAEEIE 2013), pp. 121-127.

14. K.-F. Tseng, Y.-H. Hsion, B.-J. Lwo, and C.-H. Kao, "A Multifunctional Test Chip for Microelectronic Packaging and Its Application on RF Property Measurements," pp. 78-82, 2005.

15. C. P. Wong, "Thermal-Mechanical Enhanced HighPerformance Silicone Gels and Elastomeric Encapsulants in Microelectronic Packaging," vol. 18, no. 2, pp. 58-61, 1995. https://doi.org/10.1109/95.390302

16. C. Zweben, "Advanced Composites And Other Advanced Materials For Electronic Packaging Thermal Management," 2001.

17. J. E. Bombita, J. R. Alsado, G. N. Ortiz, and M. J. B. Enojas, "Comprehensive Measurement System for Electromechanical Relay," International Journal of Advanced Trends in Computer Science and Engineering, vol. 9, no. 1, pp. 199-204, 2020. https://doi.org/10.30534/ijatcse/2020/3591.12020 